Rev. salud pública. 10 (1):160-167, 2008

Comunicaciones Breves/Brief Communication

\title{
Percepción sobre Condiciones de Trabajo y Salud de la Población Inmigrante Colombiana en Alicante, España ${ }^{1}$
}

\section{A group of Colombian immigrants' self-perception regarding working conditions and health in Alicante, Spain}

\author{
Andrés Agudelo-Suárez ${ }^{1}{ }^{2}$, Elena Ronda-Pérez ${ }^{1}$, Diana Gil-González ${ }^{1}$ y \\ Carmen Vives-Cases ${ }^{1}$
}

1 Área de Medicina Preventiva y Salud Pública. Universidad de Alicante, España. agudeloandres@odontologia.udea.edu.co, elena.ronda@ua.es, diana.gil@ua.es, carmen.vives@ua.es

2 Facultad de Odontología. Universidad de Antioquia. Medellín, Colombia.

Recibido 1 Octubre 2007/Enviado para Modificación 29 Diciembre 2007/Aceptado 15 Enero 2008

\section{RESUMEN}

Objetivo Explorar la autopercepción sobre condiciones laborales y salud en inmigrantes colombianos en Alicante, España.

Material y método Estudio cualitativo descriptivo mediante 11 entrevistas y 2 grupos de discusión en trabajadores con y sin permiso de trabajo y residencia de más de 6 meses en la provincia de Alicante (España), durante los meses de noviembre de 2006 a enero de 2007. Se realizó análisis narrativo de contenido y se obtuvieron categorías mixtas de acuerdo y disenso.

Resultados La inmigración es entendida como un proyecto para mejorar las condiciones socioeconómicas del trabajador inmigrante y su familia. El reconocimiento social y laboral puede evitar la aparición de fenómenos de exclusión y discriminación. Las personas entrevistadas asociaron sus problemas de salud con sus condiciones de vida, manifestando que constituyen limitaciones para la realización de actividades cotidianas.

Conclusiones La situación laboral y de salud es considerada desde una perspectiva multidimensional, asociada a condiciones biológicas y socioculturales. Sería necesaria una mayor acción política para mejorar la situación económica, laboral y de salud de la población inmigrante.

Palabras Clave: Población, condiciones de trabajo, salud, autopercepción (fuente: DeCS, BIREME).

\footnotetext{
${ }^{1}$ Proyecto Inmigración, Trabajo y Salud (ITSAL). Trabajo coordinado por las Universidades Pompeu Fabra (Barcelona), Universidad de Huelva, Universidad de Valencia, Instituto Sindical Trabajo, ambiente y salud (ISTAS- Madrid) y Universidad de Alicante.
} 


\section{ABSTRACT}

Objective Exploring the self-perceptions of a group of migrants from Colombia living in Alicante, Spain, regarding their working conditions and health.

Material and methods This was a qualitative and descriptive research was conducted on a group of Colombian workers (with and without legal permission to work) having lived in Alicante (Spain) for more than 6 months. 11 interviews were carried out, plus 2 focal groups, from November 2006 to January 2007. The interviews were recorded and transcribed. A narrative analysis of the contents was carried out, a mixture of categories being obtained from different viewpoints.

Results Immigration was understood as being an action for improving an immigrant worker and family's socioeconomic conditions. Work and social recognition should lead to avoiding exclusion and discrimination. The people interviewed had associated their health problems with their living conditions. Such problems were considered to be a limitation on carrying out their daily activities.

Conclusions: Working and health situations were perceived from a multi-dimensional perspective associated with biological and socio-cultural conditions. More political action should be taken for improving immigrant people's economic, work and health conditions.

Key Words: Migrant Workers, Community Workers, health, self-perception (source: $\mathrm{MeSH}, \mathrm{NLM})$.

$\mathrm{E}$ spaña es uno de los países con mayor afluencia de inmigrantes colombianos. Según la Encuesta Nacional de Demografía y Salud de Colombia en 2005, un $18 \%$ de las familias que tienen uno o más miembros residentes fuera del país están en España, principalmente por motivos económicos y laborales (1).

Se estima que en la provincia de Alicante (España) residen 19859 personas procedentes de Colombia (2). En la Comunidad Autónoma Valenciana, la población trabajadora colombiana afiliada a la seguridad social representa el 13,9 \% de toda la población trabajadora colombiana inmigrante en España (3). Las ocupaciones más habituales son para los hombres la hostelería y la construcción y para las mujeres la hostelería y el servicio doméstico $(2,4)$.

Los estudio relacionados con la salud en población inmigrante se centran en la existencia de enfermedades infecciosas relacionadas al país emisor (56), las altas hospitalarias (7), el impacto sobre la salud de condiciones socioeconómicas (8-9), y el acceso a los sistemas de atención sanitaria (10). La relación entre las condiciones de trabajo y la salud de la población inmigrante constituye un objetivo para la salud pública y la salud laboral que sin embargo no ha sido suficientemente explorado. Entre las metodologías útiles para abordar 
el fenómeno se encuentran los estudios cualitativos, que permiten explorar el proceso migratorio y las condiciones de vida de la población inmigrante a través de sus discursos. En esta línea, el objetivo del presente estudio es explorar la autopercepción acerca de las condiciones de trabajo y salud en la población inmigrante colombiana en la provincia de Alicante.

\section{MATERIAL Y MÉTODOS}

Se realizó un estudio cualitativo descriptivo contando con población colombiana trabajadora residente en Alicante más de 6 meses, con o sin permiso de trabajo y residencia. Se contactó con la población participante a través de las principales organizaciones de inmigrantes de la provincia, y de otros participantes. El trabajo de campo fue llevado a cabo entre noviembre de 2006 a enero de 2007.

Se realizaron 11 entrevistas semiestructuradas, y dos grupos de discusión, para los que se utilizó una guía que contenía aspectos relacionados con el proceso migratorio, el proceso de vinculación laboral y regularización, la historia laboral, la percepción de sus condiciones laborales y de salud, los riesgos laborales y las expectativas de futuro. Se profundizó en las respuestas de acuerdo a los objetivos del estudio. El tamaño final fue definido por saturación, al constatar que no se aportaban datos nuevos a los objetivos del estudio.

Todo el contenido fue grabado y trascrito. Se segmentaron los datos manualmente, y se atribuyeron significados para posteriormente hacer un análisis narrativo de contenido. Esto permitió la generación de categorías mixtas; aquellas establecidas inicialmente por el grupo investigador y otras emergentes propias del discurso de cada participante. Se contrastaron los datos encontrados con diferentes fuentes de información (censos, fuentes bibliográficas), y mediante la discusión y retroalimentación de los investigadores y participantes del proceso. Los resultados se presentan teniendo en cuenta las claves: grupo de discusión (G1-G2) y sexo de los participantes; para el caso de las entrevistas, se identifica el número de la misma (E1, E2,...) y el sexo. Los participantes del estudio se describen en la Tabla 1 . Se solicitó el consentimiento por escrito a las personas que voluntariamente participaron en la investigación, garantizando confidencialidad y respeto de la información.

\section{RESULTADOS}

Determinantes de acceso al mercado laboral Para la población entrevistada, emigrar es una forma de mejorar las condiciones 
laborales y económicas para el sostenimiento de sus familias. Como determinantes de este proceso, se identificaron el tipo de migración (económica, política, académica, o personal), requerimientos de visado, experiencias de migraciones previas en otros países (Francia, Venezuela o Estados Unidos) y el disponer de recursos económicos para viajar.

"Yo llevo aquí 5 años y medio, también nos vinimos por la situación económica, el trabajo de nosotros se puso difícil, se puso como mala la cosa y los dos una quincena sin pagarnos el sueldo y los dos llevados" [G2: Mujeres]

Tabla 1. Descripción de los participantes

\begin{tabular}{|c|c|c|c|c|}
\hline Descripcion & Clave & Sexo & $\begin{array}{c}\text { Situación } \\
\text { legal }\end{array}$ & Actividad laboral \\
\hline Grupo de discusión $1[4]^{*}$ & G1 & Hombres & Regular & $\begin{array}{l}\text { Hosteleria, } \\
\text { Servicio Doméstico, } \\
\text { Industria, Construcción }\end{array}$ \\
\hline Grupo de discusión 2 [6] * & $\mathrm{G} 2$ & Mujeres & Regular & $\begin{array}{l}\text { Hosteleria, } \\
\text { Servicio Doméstico }\end{array}$ \\
\hline Entrevista 1 & E1 & Hombre & Regular & $\begin{array}{l}\text { Hosteleria, } \\
\text { Venta inmobiliaria }\end{array}$ \\
\hline Entrevista 2 & E2 & Hombre & Regular & $\begin{array}{l}\text { Hosteleria, } \\
\text { Dirección y administración }\end{array}$ \\
\hline Entrevista 3 & E3 & Mujer & Regular & Administración \\
\hline Entrevista 4 & E4 & Mujer & Regular & Hosteleria \\
\hline Entrevista 5 & E5 & Hombre & Regular & Hosteleria \\
\hline Entrevista 6 & E6 & Mujer & Regular & Servicio Doméstico \\
\hline Entrevista 7 & E7 & Hombre & Regular & Seguros \\
\hline Entrevista 8 & E8 & Mujer & Irregular & Servicio Doméstico \\
\hline Entrevista 9 & E9 & Hombre & Regular & Hosteleria \\
\hline Entrevista 10 & E10 & Mujer & Regular & Hosteleria \\
\hline Entrevista 11 & E11 & Hombre & Irregular & Construcción \\
\hline
\end{tabular}

* Número de participantes en el grupo de discusión

El trabajo en Colombia se percibe como inestable, de bajo nivel salarial y con segregación y discriminación por la edad. En la actualidad no trabajan en oficios relacionados con su profesión, en parte por los trámites para la homologación de títulos. La insatisfacción laboral es compensada por factores de tipo económico.

(...) "Sabemos que en Colombia hay muchos chicos profesionales y que están ganando muchísimo menos de lo que aquí podemos ganar cuidando un niño o cuidando un abuelo y a veces es el factor económico el que nos lleva a trabajar" (...) "Pero lo positivo es que hay trabajo, es que estamos ganando dinero, o sea, emocionalmente eso nos golpea muchísimo, pero también es un aliciente cuando uno recibe el dinero... [G2: Mujeres].

La población inmigrante colombiana percibe más competencia laboral, por la presencia de trabajadores de otras nacionalidades, aspecto que para ellos influiría en su contratación y en la disminución de beneficios sociales. 
"A nosotros nos toca capacitarnos, evolucionar, si nosotros no queremos recibir una presión indebida de inmigración adicional que nos pueden quitar el trabajo como a muchos se lo estánquitando porque consiguen un africano, qué están haciendo ahora... están consiguiendo africanos sin papeles y el africano trabaja todo el día sin parar" [G1: Hombres]

Se ha expresado la percepción de los estereotipos de personas procedentes de Colombia, manifestando además experiencias de discriminación. No obstante, la ganancia de espacios de inserción laboral parece generar oportunidades de reconocimiento y respeto a su condición inmigrante.

"Yo me he encontrado con llegar a un sitio y reparar una alarma, y decirme: ¿Usted de dónde es? Y digo yo: colombiano porque nunca lo he negado y me dicen: pues no, no quiero que usted me revise mi alarma, ya llamaré a otra empresa para que manden a otra persona" [G1: Hombres]

(...) ese es un trabajo muy personal que cada una se va haciendo y que dado el entorno en el que nos estamos desenvolviendo ya no es la colombiana, es (...), es (...) y es ella la que crea su propia identidad. [G2: Mujeres]

La opinión sobre redes sociales como las asociaciones de inmigrantes se percibe elemental, por que prestan ayuda en caso de problemas laborales. Sin embargo, se evidencia baja participación en asociaciones y sindicatos.

"Regularmente yo puedo afirmar que los colombianos tenemos poca visión sobre el asociacionismo (...) si la gente se asocia mucho en esos lugares, creo que no, entonces les cuesta mucho asociarse, pero una vez descubres la labor que tú haces aquí entonces la gente ya está viniendo a asociarse porque saben que están bien asesorados, bien informados y pueden hacer valer sus derechos y cumplir sus obligaciones" [E3: Mujer]

Se presentan situaciones de incumplimiento de las condiciones de trabajo inicialmente pactadas, entre empleadores y empleados tanto de pequeñas como medianas empresas de los primeros inmigrantes que llegaron al territorio.

"...Es que de cierta forma los colombianos aquí como están súper bien muchas veces por debajean a sus empleados, porque hombre (...) ellos por ahorrarse dinero dejan gente trabajando más de las horas que son y solo van a pagar media seguridad social, eso no es normal, es más con sus coterráneos..." [E1: Hombre]

\section{Estado de Salud}

La percepción de estado de salud está relacionada principalmente con las condiciones de vida. La enfermedad se percibe como tal cuando impide el cumplimiento de las actividades cotidianas, entre ellas el trabajo. Se mencionan enfermedades de adaptación como las infecciones respiratorias, trastornos alimentarios, alergias, entre otras. También refieren signos y síntomas 
relacionados con el tipo de trabajo que realizan, como lo son las lumbalgia o problemas articulares.

"La salud de una persona cuando se va a otro país, en el caso nuestro de los latinos, se puede desmejorar por lo que vienes a desarrollar trabajos que no eran habituales a lo que se hacía, algunos vienen a hacer lo que hacían allí pero cuando la persona cambia de trabajo en general eso le afecta, tanto su estado anímico como emocional y su salud física entonces claro, se nota que se desmejora la salud" [E9: Hombre]

Las personas entrevistadas identifican los factores de riesgo relacionados con la actividad laboral que desempeñan. Sin embargo en algunos casos, perciben como normal está situación. Se evidencia falta de formación en prevención de riesgos laborales.

"Hay muchos riesgos, sobre todo muchas enfermedades posteriores, por ejemplo el hecho de que estés en una cocina con una temperatura posterior a 50 grados, llegar a manejar esa temperatura dentro de una cocina y que tu te tengas que ir a meter a un cuarto frío o abrir nevera, congelador, cualquier cosa de esas, eso va afectar tu salud en un futuro" [E1: Hombre]

"...en la construcción pues riesgos de problemas en la columna por la postura" [E11: Hombre]

También se manifiesta en los discursos la condición propia del síndrome del inmigrante, con síntomas como el estrés, el cambio de clima, el desarraigo, sentimientos de añoranza de las costumbres propias del país de origen, depresión y angustia.

"... es el síndrome del inmigrante, o sea, psicológicamente nosotros nos afectamos muchísimo, es que el desarraigo, el cambio de clima, estas estaciones tan fuertes, nosotros no estamos preparados para eso, y eso incide y nos dan unos bajones muy fuertes y nos afecta muchísimo la salud física." [G2: Mujeres]

\section{DISCUSIÓN}

Los principales problemas de salud manifestados por la población inmigrante colombina entrevistada para este estudio tiene tienen que ver con sus condiciones de vida y con las características del trabajo que realizan.

Los y las participantes conforman una historia laboral amplia, un nivel educativo alto y emigran principalmente por motivos económicos y políticos (11). En el proceso migratorio se visualiza la importancia de las redes de apoyo (familiares, amigos e instituciones) y de los primeros inmigrantes que iniciaron el ciclo -los primo-inmigrantes (12). Esto es un elemento fundamental para la inserción del trabajador en la dinámica socio-cultural del nuevo país (13), y para hacer más atractivo el país para otros inmigrantes. 
Se ha identificado en los discursos la importancia de los problemas de salud relacionados con la adaptación al nuevo contexto geográfico, así como con las condiciones biológicas y personales $(14,15)$. Por otra parte, no se manifestó en los discursos de la población inmigrante la existencia enfermedades que pudieran ser diferentes a los de la población autóctona. En general se observa en sus discursos un nivel de salud aceptable para cumplir con sus actividades familiares, personales y laborales.

El concepto de salud se considera multidimensional, y se asocia a diferentes determinantes sociales y culturales como la educación, la edad, el género, la cultura sanitaria, el medio ambiente, y los factores biológicos (15), así como a aspectos vinculados con el país de origen (16). Es por ello que el análisis del contexto, por ejemplo el económico y laboral, es fundamental para identificar la exposición de la población inmigrante a posibles riesgos laborales en mayor medida que la población autóctona (17).

El "síndrome del inmigrante", definido como síndrome de Ulises, se percibe asociado a estrés crónico (18), siendo propio de los duelos que ellos y ellas establecen al encontrarse en una cultura diferente, $\mathrm{y}$ al hecho de padecer en algunos casos, insatisfacción y temporalidad laboral, alta rotación de puestos de trabajo, y sentimientos de marginalidad y exclusión (19).

El incremento de la participación de la población inmigrante en espacios sociales y laborales podría contribuir a una mayor percepción de los derechos y del bienestar en el país de destino. Para ello sería fundamental un mayor impulso de la acción política orientada a mejorar su situación económica, laboral y de salud, fomentando así la integración laboral y social en el mercado de trabajo español entre todos los grupos de población *

Agradecimientos. Los autores agradecen la participación de las personas que colaboraron en las entrevistas y en los grupos de discusión con sus aportes y discursos, así como las contribuciones intelectuales de Mercedes Carrasco Portiño, candidata a $\mathrm{PhD}$ en Salud Pública de la Universidad de Alicante. Financiación: Consellería de Empresa, Universidad y Ciencia. Generalitat Valenciana [AE/ 07/068].

\section{REFERENCIAS}

1. Profamilia. Salud sexual y reproductiva en Colombia: resultados encuesta nacional de demografía y salud 2005. Bogotá: Profamilia; 2005.

2. Centro de estudios para la integración social y formación de inmigrantes. Fundación de la Comunidad Valenciana. Miradas sobre la Inmigración 12: Ciudadanos del Mundociudadanos de Colombia. [Internet] Disponible en: http://www.ceim.net Consultado 8 denoviembre de 2006. 
3. Ministerio de trabajo y asuntos sociales. Trabajadores extranjeros afiliados en alta laboral. [Internet] Disponible en http://www.seg-social.es/inicio/ Consultado 10 de abril de 2007

4. Instituto Nacional de Estadística. Censo de población y vivienda 2001. [Internet] Disponible en: http://www.ine.es Consultado 8 de Octubre de 2006.

5. Ramos JM, Pastor C, Masía M, Cascales E, Royo C, Gutiérrez F. Examen de salud en la población inmigrante: prevalencia de infección tuberculosa latente, hepatitis B, hepatitis C, infección por el VIH y sífilis. Enfermedades Infecciosas y Microbiología Clínica. 2003; 21(10): 540-542.

6. Lacalle M, Gil G, Sagardui J, González E, Martínez R, Orden B. Resultados de la aplicación de un examen de salud en población inmigrante. Aten Primaria 2000; 25: 634-638.

7. Salazar A, Navarro E, Abad I et al. Diagnósticos al alta hospitalaria de las personas inmigrantes de la ciudad de Valencia 2001-2002. Rev Española Salud Pública 2003; 77(6): 713-723.

8. Benítez RT, Llerena AP, López GR, Brugera MC, Lasheras L. Determinantes socioeconómicos en un colectivo de familias inmigrantes. An Pediatr (Barc) 2004; 60(1):9-15.

9. Esteban MM. Motivos de consulta y características demográficas de una comunidad de inmigrantes "sin papeles" en el distrito de Usera-Villaverde (Madrid). Aten Primaria, 2001;27:25-28.

10. Ramos M, García R, Prieto MA, March JC. Problemas y propuestas de mejora en la atención sanitaria a los inmigrantes económicos. Gac Sanit 2001; 15(4): 320-326.

11. Hofmann A, Escala Z. The Colombian community in Metropolitan New York Migration World Magazine, 1999; 27 (4): 13-17.

12. Arango J. La inmigración en España a comienzos del siglo XXI. En: Maldonado J. Informe sobre la situación demográfica en España. España: Fundación Fernando Abril Martorell; 2004. pp 161-186.

13. Bermúdez E. Imágenes de salud y enfermedad de las mujeres colombianas inmigrantes en España. Revista Gerencia y Políticas de Salud, 2004; 3(7): 78-100.

14. Jansá JM. Inmigración extranjera en el estado español Consideraciones desde la Salud Pública. Rev Española de Salud Pública, 1998; 72 (3): 165-168.

15. Fuertes C, Martín Laso MA. El inmigrante en la consulta de atención primaria. An Sist Sanit Navar. 2006; 29 (Supl 1): 9-25.

16. Jansá JM, García de Olalla P. Salud e inmigración: nuevas realidades y nuevos retos. Gac Sanit, 2004; 18(Supl): 207-13

17. Ahonen EQ, Benavides FG. Risk of fatal and no fatal occupational injury in foreign workers in Spain. JEpidemiol Community Health 2006; 60: 424-426.

18. Achotegui J. 2004. Emigrar en situación extrema. El Síndrome del Inmigrante con estrés crónico y múltiple. Síndrome de Ulises. Revista Norte de Salud Mental. 2004; 5 (21):39-53.

19. García J, Sanz C. Salud mental en inmigrantes: el nuevo desafío. Med Clin (Barc) 2002; 118(5):187-191. 